\title{
Multi-source operator channels: Efficient capacity-achieving codes
}

\author{
Hongyi Yao*, Theodoros K. Dikaliotis ${ }^{\dagger}$, Sidharth Jaggi ${ }^{\ddagger}$, Tracey $\mathrm{Ho}^{\dagger}$ \\ *Tsinghua University, Beijing, China. Email: yaohongyi03@gmail.com \\ ${ }^{\dagger}$ California Institute of Technology, Pasadena, CA, USA. Email: \{tdikal, tho $\} @$ caltech.edu \\ ${ }^{\ddagger}$ Chinese University of Hong Kong. Email: jaggi@ie.cuhk.edu.hk
}

\begin{abstract}
The network communication scenario where one or more receivers request all the information transmitted by different sources is considered. We introduce the first polynomialtime (in network size) network codes that achieve any point inside the rate-region for the problem of multiple-source multicast in the presence of malicious errors, for any fixed number of sources. Our codes are fully distributed and different sources require no knowledge of the data transmitted by their peers. Our codes are "end-to-end", that is, all nodes apart from the sources and the receivers are oblivious to the adversaries present in the network and simply implement random linear network coding.
\end{abstract}

\section{INTRODUCTION}

Information dissemination can be optimized with the use of network coding since it maximizes the network throughput in multicast transmission scenarios [1]. At the same time network coding is highly vulnerable to malicious attacks from rogue users. The presence of even a small number of adversarial nodes can contaminate the majority of packets in a network, preventing receivers from decoding.

For both coherent (when the network transform is known a priori to the receiver(s)) and non-coherent (when no such information is known a priori to the receiver(s)) cases, we consider the design of multisource network error-correcting codes that are resilient against worst-case network errors, i.e., against errors injected by computationally unbounded adversaries who know the network topology, the coding scheme and messages used by the sources, all local encoding coefficients at the internal nodes and the decoding schemes employed by the receivers. Although the single source network error-correcting codes are well studied, the multisource scenario still faces the following challenge:

Challenge: The single source network error-correcting codes require the source to judiciously insert redundancy into the transmitted codeword. However, in the distributed multisource case, all sources need to work cooperatively to insert redundancy for their messages. Thus, direct implementations of single-source error-correcting codes (for instance [2]) fail to achieve optimal rates.

\section{A. Related works}

For the single source multicast scenario, the work of CaiYeung [3] first studied the network error-correcting problem, and their scheme requires high (exponential in the network size) design complexity. Further works by [4], [5], [6] provided network error-correcting codes with design and implementation complexity that is low (i.e., polynomial in size of the network parameters).

For the multisource multicast scenario without network errors, the works [7], [8] and [9] achieve any point inside the rate region. For the multisource multicast scenario with worst-case network errors, the work in [10] gives the capacity region for the problem, but the achievability proof uses codes with high decoding complexity (exponential in network size). To be concrete, let $\left\{\mathcal{S}_{1}, \mathcal{S}_{2}, \ldots, \mathcal{S}_{s}\right\}$ be the sources and $z$ be the number of network edge errors. A rate tuple $\left(R_{1}, R_{2}, \ldots, R_{s}\right)$ is achievable if and only if for any subset $\mathcal{I}$ of $\{1,2, \ldots, s\}, \sum_{i \in \mathcal{I}} R_{i} \leq C_{\mathcal{I}}-2 z$, where $C_{\mathcal{I}}$ is the capacity from $\left\{\mathcal{S}_{i}: i \in \mathcal{I}\right\}$ to the receivers ${ }^{1}$. The work [2] provides efficient error-correcting code construction for linear subspace codes with subspace injection/deletion errors. For the edge rank error model ${ }^{2}$ considered in this paper, using the technique in [2] a strict subregion is attainable. That is, [2] achieves rate tuple $\left(R_{1}, R_{2}, \ldots, R_{s}\right)$ when $\sum_{i \in \mathcal{I}} R_{i} \leq C_{\mathcal{I}}-2|\mathcal{I}| z$ for each $\mathcal{I} \subseteq\{1,2, \ldots, s\}$.

\section{B. Our contributions}

For both coherent and non-coherent cases, we provide the first efficient algorithm achieving the whole rate-region demonstrated in [10], via a novel "multiple field-extension" technique. This technique is crucial - direct implementations of previous low complexity network error correction codes (as in [2]) do not achieve the full rate-region.

Our codes have implementation complexity that is polynomial in the size of the network. Furthermore our codes are fully distributed in the sense that different sources require no knowledge of the data transmitted by their peers and end-toend, i.e., all nodes are oblivious to the adversaries present in the network and simply implement random linear network coding [11].

In Section II we formulate the problem and introduce the mathematical preliminaries. The code constructions for coherent case and non-coherent cases are provided in Section III and Section IV respectively.

\footnotetext{
${ }^{1}$ Let $\left\{\mathcal{R}_{1}, \mathcal{R}_{2}, \ldots, \mathcal{R}_{d}\right\}$ be the receivers, the capacity from $\left\{\mathcal{S}_{i}: i \in \mathcal{I}\right\}$ to the receivers is defined as $\min _{j \in[1, d]}\left(\max -\right.$ flow $\left.\left(\left\{\mathcal{S}_{i}: i \in \mathcal{I}\right\}, \mathcal{R}_{j}\right)\right)$.

${ }^{2}$ More details about subspace injection/deletion errors and edge rank errors can be found in [6].
} 


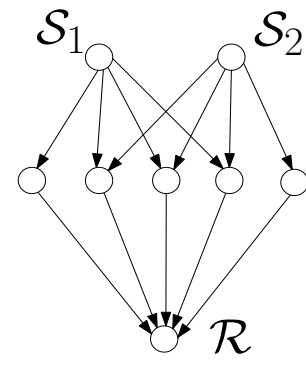

(a) Example Network

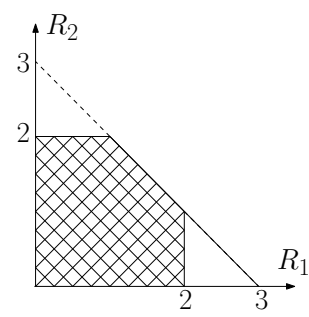

(b) Rate Region
Fig. 1. An example network with two sources. The Network in Figure 1(a) has $C_{1}=C_{2}=4, C=5$ and the adversary can inject $z=1$ error packet. The achievable rate region is shown in the dark region of Figure 1(b).

\section{PRELIMINARIES}

\section{A. Model}

We consider a delay-free network $\mathcal{G}=(\mathcal{V}, \mathcal{E})$ where $\mathcal{V}$ is the set of nodes and $\mathcal{E}$ is the set of edges. The capacity of each edge is normalized to be one symbol of $\mathbb{F}_{p}$ per unit time. Edges with non-unit capacity are modeled as parallel edges.

For notational convenience we restrict ourselves to the analysis of the situation where there are only two sources $\mathcal{S}_{1}, \mathcal{S}_{2} \in \mathcal{V}$ transmitting information to one receiver $\mathcal{R} \in \mathcal{V}$, since the extension of our results to more sources and receivers is straightforward. The minimum cut capacity from source $\mathcal{S}_{i}$ to $\mathcal{R}$ is denoted by $C_{i}$ for $i \in[1,2]$, and the minimum cut capacity from both sources to the receiver is equal to $C$.

Within the network there is a hidden adversary trying to interfere with the transmission of information by observing all the transmissions in the network and injecting its own packets in any $z \operatorname{links}^{3}$, that may be chosen as a function of his complete knowledge of the network, the message, and the communication scheme.

The sources on the other hand know nothing about each other's transmitted information and the links compromised by the adversary. Their goal is to add redundancy into their transmitted packets so that they can achieve any rate-tuple $\left(R_{1}, R_{2}\right)$ such that $R_{1} \leq C_{1}-2 z, R_{2} \leq C_{2}-2 z$, and $R_{1}+R_{2} \leq C-2 z$ (this is the rate region of the multi-source multicast problem proved in [10]). An example network and its rate region is shown in Figure 1.

To simplify the discussion we show the code construction for rate-tuple $\left(R_{1}, R_{2}\right)$ satisfying $R_{1} \leq C_{1}-2 z, R_{2} \leq C_{2}-2 z$, $R_{1}+R_{2}+2 z=C$ and exactly $C$ edges reach the receiver $\mathcal{R}$ (if more do, redundant information can be discarded).

\section{B. Random linear network coding}

In this paper, we consider the following well-known distributed random linear coding scheme [11].

Sources: The source $\mathcal{S}_{i}$ arranges the data into a $R_{i} \times \ell$ Message matrix $M_{i}$ over $\mathbb{F}_{p}$ (here the packet-length $\ell$ is a network design parameter). For $i \in[1,2]$ source $\mathcal{S}_{i}$ then takes

\footnotetext{
${ }^{3}$ Note that since each transmitted symbol in the network is from a finite field, modifying symbol $x$ to symbol $y$ is equivalent to injecting/adding symbol $y-x$ into $x$.
}

independent and uniformly random linear combinations over $\mathbb{F}_{p}$ of the rows of $M_{i}$ to generate respectively the packets transmitted on each outgoing edge.

Network nodes: Each internal node similarly takes uniformly random linear combinations of the packets on incoming edges to generate packets transmitted on outgoing edges.

Receiver: The receiver $\mathcal{R}$ constructs the $C \times \ell$ matrix $Y$ over $\mathbb{F}_{p}$ by treating the received packets as consecutive length$\ell$ row vectors of $Y$ (recall that exactly $C$ edges reach $\mathcal{R}$ ). In the case that the network is error-free the network's internal linear operations induce linear transforms from $M_{i}$ to $Y$ as

$$
Y=T_{1} M_{1}+T_{2} M_{2},
$$

where $T_{i}$ is the overall transform matrix from $\mathcal{S}_{i}$ to $\mathcal{R}$.

\section{Finite field extension}

We denote by $\mathbb{F}_{p}^{m \times n}$ the set of all $m \times n$ matrices with elements from $\mathbb{F}_{p}$. The $m$-dimensional identity matrix is denoted by $I_{m}$, and the zero matrix of any dimensions is denoted by $O$. Vectors are in bold-face (e.g. A).

We first review some finite field theory. Every finite field $\mathbb{F}_{p}$, where $p$ is a prime power, can be algebraically extended ${ }^{4}$ [12] to a larger finite field $\mathbb{F}_{q}$, where $q=p^{n}$ for any positive integer $n$. Since $\mathbb{F}_{q}$ includes $\mathbb{F}_{p}$ as a subfield thus any matrix $A \in \mathbb{F}_{p}^{m \times \ell}$ is also a matrix in $\mathbb{F}_{q}^{m \times \ell}$. Hence throughout the paper matrix multiplication over different fields (one over the base field and the other from the extended field) is allowed and computed over the extended field.

A bijective map from $\mathbb{F}_{p}^{m \times n}$ to $\mathbb{F}_{q}^{m}$ is defined as follows:

- For each $A \in \mathbb{F}_{p}^{m \times n}$, the folded version of $A$ is a vector $\mathbf{A}^{f}$ in $\mathbb{F}_{q}^{m}$ given by $A \mathbf{a}^{\mathrm{T}}$ where $\mathbf{a}=\left\{a_{1}, \ldots, a_{n}\right\}$ is a basis of the extension field $\mathbb{F}_{q}$ with respect to $\mathbb{F}_{p}$. Here we treat the $i^{\text {th }}$ row of $A$ as a single element in $\mathbb{F}_{q}$ to obtain the $i^{\text {th }}$ element of $\mathbf{A}^{f}$. For instance let $A=\left[\begin{array}{ll}1 & 0 \\ 1 & 1\end{array}\right]$ be a matrix in $\mathbb{F}_{2}^{2 \times 2}$. Then the operation of folding it into $\mathbb{F}_{4}^{2}$ gives $\mathbf{A}^{f}=\left[\begin{array}{l}(1,0) \\ (1,1)\end{array}\right]=\left[\begin{array}{l}2 \\ 3\end{array}\right] \in \mathbb{F}_{4}^{2}$ (where $2 \equiv x$ and $\left.3 \equiv x+1 \bmod \left(x^{2}+x+1\right)[12]\right)$.

- For each $\mathbf{B} \in \mathbb{F}_{q}^{m}$, the unfolded version of $\mathbf{B}$ is a matrix $B^{u}$ in $\mathbb{F}_{p}^{m \times n}$. Here we treat the $i^{\text {th }}$ element of $\mathbf{B}$ as a row in $\mathbb{F}_{p}^{1 \times n}$ to obtain the $i^{\text {th }}$ row of $B^{u}$. For instance let $\mathbf{B}=\left[\begin{array}{l}2 \\ 3\end{array}\right]$ be a vector in $\mathbb{F}_{4}^{2}$. Then the operation of unfolding it into $\mathbb{F}_{2}^{2 \times 2}$ gives $B^{u}=\left[\begin{array}{ll}1 & 0 \\ 1 & 1\end{array}\right]$.

We can also extend these operations to include more general scenarios. Specifically any matrix $A \in \mathbb{F}_{p}^{m \times \ell n}$ can be written as a concatenation of matrices $A=\left[A_{1} \ldots A_{\ell}\right]$, where $A_{i} \in F_{p}^{m \times n}$. The folding operation is defined as follows: $A^{f}=\left[\mathbf{A}_{1}^{f} \ldots \mathbf{A}_{\ell}^{f}\right]$. Similarly the unfolding operation $u$ can be applied to a number of submatrices of a large matrix, e.g., $\left[\mathbf{A}_{1}^{f} \ldots \mathbf{A}_{\ell}^{f}\right]^{u}=\left[\left(\mathbf{A}_{1}^{f}\right)^{u} \ldots\left(\mathbf{A}_{\ell}^{f}\right)^{u}\right]=\left[A_{1} \ldots A_{\ell}\right]$.

\footnotetext{
${ }^{4}$ Let $\mathbb{F}_{p}[x]$ be the set of all polynomials over $\mathbb{F}_{p}$ and $f(x) \in \mathbb{F}_{p}[x]$ be an irreducible polynomial of degree $n$. Then $\mathbb{F}_{p}[x] / f(x)$ defines an algebraic extension field $\mathbb{F}_{p^{n}}$ by a homomorphism mapping [12].
} 
TABLE I

SUMMARY OF FIELD NOTATIONS

\begin{tabular}{cccc}
\hline \hline Field & $\mathbb{F}_{p}$ & $\mathbb{F}_{q}$ & $\mathbb{F}_{Q}$ \\
\hline Size & $p$ & $q=p^{n}$ & $Q=q^{N}$ \\
\hline
\end{tabular}

In the paper double algebraic extensions are considered. More precisely let $\mathbb{F}_{Q}$ be an algebraic extension of $\mathbb{F}_{q}$, where $Q=q^{N}=p^{n N}$ for any positive integer $N$. Table I summarize the notation of the fields considered.

Note: Of the three fields $\mathbb{F}_{p}, \mathbb{F}_{q}$ and $\mathbb{F}_{Q}$ defined above, two or sometimes all three appear simultaneously in the same equation. To avoid confusion, unless otherwise specified, the superscript $f$ for folding is from $\mathbb{F}_{p}$ to $\mathbb{F}_{q}$, and the superscript $u$ for unfolding is from $\mathbb{F}_{q}$ (or $\mathbb{F}_{Q}$ ) to $\mathbb{F}_{p}$.

\section{Row-space distance}

For any matrices $B_{1} \in \mathbb{F}_{p}^{m_{1} \times n}$ and $B_{2} \in \mathbb{F}_{p}^{m_{2} \times n}$ let $\mathcal{B}_{1}$ and $\mathcal{B}_{2}$ be the subspaces spanned by the rows of $B_{1}$ and the rows of $B_{2}$ respectively. The row-space distance of $B_{1}$ and $B_{2}$ (which is a metric and satisfies the triangle inequality [13].) is defined as $d_{S}\left(B_{1}, B_{2}\right)=\operatorname{dim}\left(\operatorname{span}\left(\mathcal{B}_{1} \cup \mathcal{B}_{2}\right)\right)-\operatorname{dim}\left(\operatorname{span}\left(\mathcal{B}_{1} \cap \mathcal{B}_{2}\right)\right)$. If $m_{1}=m_{2}=m$, Proposition 1 below is a direct consequence of Corollary 3 in [5]:

Proposition 1. $d_{S}\left(B_{1}, B_{2}\right) \leq 2 \operatorname{rank}\left(B_{1}-B_{2}\right)$.

\section{E. Gabidulin codes}

Gabidulin in [14] introduced a class of error correcting codes over $\mathbb{F}_{p}^{m \times n}$. Let $\mathbf{X} \in \mathbb{F}_{q}^{R}$ be the information vector, $G \in \mathbb{F}_{q}^{m \times R}$ be the generator matrix, $(G \mathbf{X})^{u} \in \mathbb{F}_{p}^{m \times n}$ be the transmitted matrix, $Z \in \mathbb{F}_{p}^{m \times n}$ be the error matrix, and $(G \mathbf{X})^{u}+Z \in \mathbb{F}_{p}^{m \times n}$ be the received matrix. Then decoding is possible if and only if $\operatorname{rank}(Z) \leq\left\lfloor\frac{d}{2}\right\rfloor$, where $d=m-R+1$ is the minimum distance of the code.

The work of [5] utilizes the results of [14] to obtain network error-correcting codes with the following properties:

Theorem 1 (Theorem 11 in [5]). Let $Z$ be expressed as $Z=$ $\sum_{i \in[1, \tau]} \mathbf{L}_{i} \mathbf{E}_{i}$, such that:

- For each $i \in[1, \tau], \mathbf{L}_{i} \in \mathbb{F}_{p}^{m \times 1}$ and $\mathbf{E}_{i} \in \mathbb{F}_{p}^{1 \times n}$;

- For each $i \in[1, \mu], \mathbf{L}_{i}$ is known a priori by the receiver;

- For each $i \in[\mu+1, \mu+\delta], \mathbf{E}_{i}$ is known a priori by the receiver;

- $2 \tau-\mu-\delta \leq d-1$,

using Gabidulin codes the receiver can decode $\mathbf{X}$ with at most $\mathcal{O}(m n)$ operations over $\mathbb{F}_{q}$.

Thus when $\mu=\delta=0$, Theorem 1 reduces to the basic case where the receiver has no prior knowledge about $Z$.

\section{COHERENT NETWORK ERROR-CORRECTING CODES}

Coherent here means the receiver $\mathcal{R}$ knows the linear transforms from both $\mathcal{S}_{1}$ and $\mathcal{S}_{2}$, i.e., $\mathcal{R}$ knows $T_{1}$ and $T_{2}$ defined in equation (1). For instance, it is possible $T_{1}$ and $T_{2}$ to be inferred by network communications before the adversary enters the network and corrupts information. Alternatively, if centralized designed network coding is used [15], $T_{1}$ and $T_{2}$ is assumed to be known by the receiver.
While the non-coherent codes we propose are more general than the coherent codes, the description of the latter is simpler, and hence we first describe them. Under the coherent assumption the goal of the section is to construct a code attaining any rate-tuple $\left(R_{1}, R_{2}\right)$ in the rate region for our communication scenario (see section II-A for details).

Encoding: Each source $\mathcal{S}_{i}, i \in[1,2]$, has information to deliver to destination $\mathcal{R}$ and organizes this information into batches of $R_{i}$ packets. Each packet is a concatenation of $\ell=k n N$ symbols from the finite field $\mathbb{F}_{p}$, where $n=R_{1}+2 z$ and $N=R_{2}+2 z$ and $k$ is a code design parameter. For simplicity we will analyze the transmission of a single batch of packets.

The way sources encode their information packets is through the use of Gabidulin codes (see Section II-E for details). More precisely the information of $\mathcal{S}_{1}$ is a matrix $X_{1} \in \mathbb{F}_{q}^{R_{1} \times k N}$, where $\mathbb{F}_{q}$ is an algebraic extension of $\mathbb{F}_{p}$ and $q=p^{n}$ (see Section II-C for details). Before transmission $X_{1}$ is multiplied with a generator matrix, $G_{1} \in \mathbb{F}_{q}^{n \times R_{1}}$, creating $G_{1} X_{1} \in \mathbb{F}_{q}^{n \times(k N)}$ whose unfolded version $M_{1}=\left(G_{1} X_{1}\right)^{u}$ is a matrix in $\mathbb{F}_{p}^{n \times \ell}$ that is transmitted through the network using the random linear network coding defined in Section II-B.

The information of $\mathcal{S}_{2}$ is a matrix $X_{2} \in \mathbb{F}_{Q}^{R_{2} \times k}$, where $\mathbb{F}_{Q}$ is an algebraic extension of $\mathbb{F}_{q}$ and $Q=q^{N}=p^{n N}$. Before transmission $X_{2}$ is multiplied with a generator matrix, $G_{2} \in \mathbb{F}_{Q}^{N \times R_{2}}$, creating $G_{2} X_{2} \in \mathbb{F}_{Q}^{N \times k}$ whose unfolded version $M_{2}=\left(G_{2} X_{2}\right)^{u}$ over $\mathbb{F}_{p}$ is a matrix in $\mathbb{F}_{p}^{N \times \ell}$ that is transmitted through the network using the random linear network coding defined in Section II-B.

Both $G_{1}$ and $G_{2}$ are chosen as generator matrices for Gabidulin codes and have the capability of correcting errors of rank at most $z$ over $\mathbb{F}_{p}$ and $\mathbb{F}_{q}$ respectively.

Decoding: The packets reaching receiver $\mathcal{R}$ can be expressed as

$$
Y=T_{1} M_{1}+T_{2} M_{2}+E,
$$

where $Y \in \mathbb{F}_{p}^{C \times \ell}$ is the matrix formed by the packets received by $\mathcal{R}, T_{1} \in \mathbb{F}_{p}^{C \times n}, T_{2} \in \mathbb{F}_{p}^{C \times N}$ are the linear transform matrices from $\mathcal{S}_{1}$ and $\mathcal{S}_{2}$ to the receiver $\mathcal{R}$, and $E \in \mathbb{F}_{p}^{C \times \ell}$ is the error matrix induced at the receiver. Note that $\operatorname{rank}(E) \leq z$ since the adversary can inject only $z$ error packets [4].

Folding equation (2) into $\mathbb{F}_{q}$ results in:

$$
Y^{f}=\left[\begin{array}{ll}
T_{1} G_{1} & T_{2}
\end{array}\right]\left[\begin{array}{c}
X_{1} \\
M_{2}^{f}
\end{array}\right]+E^{f},
$$

where $E^{f}$ has rank at most equal to $z$ according to Lemma 1 .

\section{Lemma 1. Folding a matrix does not increase its rank.}

Proof: Let matrix $H \in \mathbb{F}_{p}^{m \times k n}$ has $\operatorname{rank}(H)=r$. Thus $H=$ $W Z$, where $Z \in \mathbb{F}_{p}^{r \times k n}$ is of full row rank and $W \in \mathbb{F}_{p}^{m \times r}$ is of full column rank. After the folding operation $H$ becomes $H^{f}=W Z^{f}$ and therefore $\operatorname{rank}\left(H^{f}\right) \leq r$.

Let $D=\left[\begin{array}{ll}T_{1} G_{1} & T_{2}\end{array}\right]$. Since $R_{1}+N=R_{1}+R_{2}+2 z=C$ (see Section II-A for details), $D$ is a $C \times C$ square matrix.

Lemma 2. Matrix $D \in \mathbb{F}_{q}^{C \times C}$ is invertible with probability at least $1-|\mathcal{E}| / p$. 
Proof: Let $\mathcal{X}$ be the set of random variables over $\mathbb{F}_{p}$ comprised of the local coding coefficients used in the random linear network code. Thus the determinant of $D$ is a polynomial $\mathbf{f}(\mathcal{X})$ over $\mathbb{F}_{q}$ of degree at most $|\mathcal{E}|$ (see Theorem 1 in [11] for details). Since the variables $\mathcal{X}$ in $\mathbf{f}(\mathcal{X})$ are evaluated over $\mathbb{F}_{p}, \mathbf{f}(\mathcal{X})$ is equivalent to a vector of polynomials $\left(f_{1}(\mathcal{X}), f_{2}(\mathcal{X}), \ldots, f_{n}(\mathcal{X})\right)$, where $f_{i}(\mathcal{X}) \in \mathbb{F}_{p}[\mathcal{X}]$ is a polynomial over $\mathbb{F}_{p}$ with variables in $\mathcal{X}$. Note that $f_{i}(\mathcal{X})$ also has degree no more than $|\mathcal{E}|$ for each $i \in[1, n]$. Thus once we prove that there exists an evaluation of $\mathcal{X}$ such that $\mathrm{f}$ is a nonzero vector over $\mathbb{F}_{p}$, we can show $D$ is invertible with probability at least $1-|\mathcal{E}| / p$ by the Schwartz-Zippel lemma [16].

Since $R_{1}+N=C$ (see Section II-A for details) and $R_{1} \leq$ $C_{1}$ and $N \leq C_{2}$, there exist $R_{1}+N$ edge-disjoint-paths: $\mathcal{P}_{1}^{1}, \mathcal{P}_{2}^{1}, \ldots, \overline{\mathcal{P}}_{R_{1}}^{1}$ from $s_{1}$ to $r$ and $\mathcal{P}_{1}^{2}, \mathcal{P}_{2}^{2}, \ldots, \mathcal{P}_{N}^{2}$ from $s_{2}$ to $r$. The variables in $\mathcal{X}$ are evaluated in the following manner:

1). Let $O$ be the zero matrix in $F_{q}^{n \times N}$. We choose the variables in $\mathcal{X}$ so that the $R_{1}$ independent rows of $\left[G_{1}, O\right] \in$ $\mathbb{F}_{q}^{n \times C}$ correspond to routing information from $s_{1}$ to $R$ via $\mathcal{P}_{1}^{1}, \ldots, \mathcal{P}_{R_{1}}^{1}$.

2). Let $\left\{\mathbf{u}_{R_{1}+1}, \mathbf{u}_{R_{1}+2}, \ldots, \mathbf{u}_{C}\right\}$ be $N$ distinct rows of the identity matrix in $\mathbb{F}_{q}^{C \times C}$ such that for each $i \in[1, N], \mathbf{u}_{R_{1}+i}$ has the element 1 located at position $R_{1}+i$. Then these $N$ vectors correspond to routing information from $s_{2}$ to $r$ via $\mathcal{P}_{1}^{2}, \mathcal{P}_{2}^{2}, \ldots, \mathcal{P}_{N}^{2}$.

Under such evaluations of the variables in $\mathcal{X}$, matrix $D$ equals $\left[\begin{array}{cc}G_{1}^{\prime} & O \\ O & I_{N}\end{array}\right]$, where $G_{1}^{\prime} \in \mathbb{F}_{q}^{R_{1} \times R_{1}}$ consists of the $R_{1}$ independent rows of $G_{1}$. Hence $\mathbf{f}$ is non-zero. Using the Schwartz-Zippel Lemma $\mathbf{f} \neq 0$ and thus $D$ is invertible with probability at least $1-|\mathcal{E}| / p$ over the choices of $\mathcal{X}$.

Hence, by multiplying Equation (3) by $D^{-1}$ the receiver gets $D^{-1} Y^{f}=\left[\begin{array}{c}X_{1} \\ M_{2}^{f}\end{array}\right]+D^{-1} E^{f}$. The last $N=R_{2}+2 z$ rows of $D^{-1} Y^{f}$ are $\left(D^{-1} Y^{f}\right)_{d}=M_{2}^{f}+\left(D^{-1} E^{f}\right)_{d}$, where the subscript $d$ stands for the last $N$ rows of each matrix.

Note: To show why $\mathcal{S}_{2}$ uses a generator matrix $G_{2}$ over a double-extended field $\mathbb{F}_{Q}=\mathbb{F}_{q^{N}}=\mathbb{F}_{p^{n N}}$, consider what happens if instead it uses $\mathbb{F}_{Q}=\mathbb{F}_{q}$. In this case the matrix $M_{2}^{f}+\left(D^{-1} E^{f}\right)_{d}$ is indeed of the form required for successful decoding of Gabidulin codes as long as $\left(D^{-1} E^{f}\right)_{d}^{u}$ has rank less than $z$ over $\mathbb{F}_{p}$. But this is not generally the case since $D^{-1}$ belongs to $\mathbb{F}_{q}$ but not $\mathbb{F}_{p}$. Therefore although $E^{f}$ and consequently $D^{-1} E^{f}$ have rank less than $z$ over $\mathbb{F}_{q}$, the rank of $\left(D^{-1} E^{f}\right)_{d}^{u}$ might increase over $\mathbb{F}_{p}$.

If source $\mathcal{S}_{2}$ uses a generator matrix $G_{2}$ defined over $\mathbb{F}_{Q}=$ $\mathbb{F}_{q^{N}}$ that is able to correct rank $z$ errors over $\mathbb{F}_{q}$, we can prove the main result in this section as follows.

Theorem 2. A coherent receiver $\mathcal{R}$ can efficiently decode both $X_{1}$ and $X_{2}$ correctly with probability at least $1-2|\mathcal{E}| / p$.

Proof: First, according to Lemma 2 matrix $D$ is invertible with probability at least $1-|\mathcal{E}| / p$. Since $G_{2}$ is able to correct rank $z$ errors over $\mathbb{F}_{q}$, using $\left(D^{-1} Y^{f}\right)_{d}=M_{2}^{f}+\left(D^{-1} E^{f}\right)_{d}, \mathcal{R}$ can execute the Gabidulin decoding algorithm and get $X_{2}$.

Second, once $X_{2}$ is known $T_{2} M_{2}$ is subtracted from $Y$ to result in $T_{1} M_{1}+E$. Since $T_{1}$ is left invertible with probability at least $1-|\mathcal{E}| / p$ (by [11]), $\mathcal{R}$ can multiply $T_{1} M_{1}+E$ with the left inverse of $T_{1}$ giving $M_{1}+T_{1}^{-1} E$. Since $\operatorname{rank}\left(T_{1}^{-1} E\right) \leq z$ over base field $\mathbb{F}_{p}$, the execution of the Gabidulin decoding algorithm results in $X_{1}$. In the end the overall probability of correct decoding is at least $1-2|\mathcal{E}| / p$.

\section{NON-COHERENT ERROR CORRECTION}

In the non-coherent case it is assumed that receiver $\mathcal{R}$ does not know the network transform matrices $T_{1}$ and $T_{2}$ of the two sources prior to communication in the presence of the adversary. Assuming a non-coherent receiver the goal of this section is to construct codes attaining any rate-tuple $\left(R_{1}, R_{2}\right)$ in the rate region for our communication scenario (see section II-A for details).

Encoding: In the scenario where the receiver $\mathcal{R}$ does not know $T_{1}$ and $T_{2}$ a priori the two sources append headers on their transmitted packets to convey information about $T_{1}$ and $T_{2}$ to the receiver. Thus source $\mathcal{S}_{1}$ constructs message matrix $\left[\begin{array}{lll}I_{n} & O & M_{1}\end{array}\right]$ with the zero matrix $O$ having dimensions $n \times N$, and source $\mathcal{S}_{2}$ constructs a message matrix $\left[\begin{array}{lll}O & I_{N} & M_{2}\end{array}\right]$ with the zero matrix $O$ having dimension $N \times n$. The identity and zero matrices have elements from $\mathbb{F}_{p}$ and the $M_{1}, M_{2}$ matrices in $\mathbb{F}_{p}^{C \times \ell}$ have the same definitions as in Section III-A.

Decoding: The two message matrices are transmitted to the receiver $\mathcal{R}$ through the network with the use of random linear network code and therefore the receiver gets:

$$
Y=\left[\begin{array}{lll}
Y_{1} & Y_{2} & Y_{3}
\end{array}\right]=\left[\begin{array}{lll}
T_{1} & T_{2} & A
\end{array}\right]+E,
$$

where $A=T_{1} M_{1}+T_{2} M_{2} \in \mathbb{F}_{p}^{C \times \ell}$ and $E \in \mathbb{F}_{p}^{C \times(n+N+m)}$ has rank no more than $z$ over field $\mathbb{F}_{p}$. Let $E=$ $\left[\begin{array}{lll}E_{1} & E_{2} & E_{3}\end{array}\right]$, where $E_{1} \in \mathbb{F}_{p}^{C \times n}$ and $E_{2} \in \mathbb{F}_{p}^{C \times N}$ and $E_{3} \in \mathbb{F}_{p}^{C \times \ell}$. As in the decoding scheme in Section III the receiver $\mathcal{R}$ first decodes $X_{2}$ and then $X_{1}$.

Stage 1: Decoding $X_{2}$ : Let $Y_{a}=\left[\begin{array}{lll}Y_{1} G_{1} & Y_{2} & Y_{3}^{f}\end{array}\right]$ be a matrix in $\mathbb{F}_{q}^{C \times\left(R_{1}+N+k N\right)}$. To be precise:

$$
Y_{a}=\left[\begin{array}{lll}
T_{1} G_{1} & T_{2} & A^{f}
\end{array}\right]+\left[\begin{array}{lll}
E_{1} G_{1} & E_{2} & E_{3}^{f}
\end{array}\right] .
$$

Receiver $\mathcal{R}$ uses invertible row operations over $\mathbb{F}_{q}$ to transform

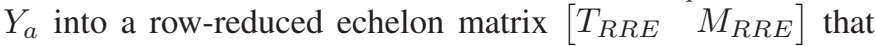
has the same row space as $Y_{a}$, where $T_{R R E}$ has $C=R_{1}+N$ columns and $M_{R R E}$ has $k N$ columns. Then the following propositions are from the results ${ }^{5}$ proved in [5]:

Proposition 2. 1) The matrix $\left[\begin{array}{ll}T_{R R E} & M_{R R E}\end{array}\right]$ takes the

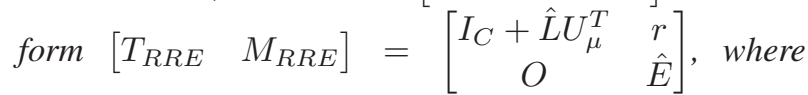
$U_{\mu} \in \mathbb{F}_{q}^{C \times \mu}$ comprises of $\mu$ distinct columns of the $C \times C$ identity matrix such that $U_{\mu}^{T} r=0$ and $U_{\mu}^{T} \hat{L}=-I_{\mu}$. In particular, $\hat{L}$ in $\mathbb{F}_{q}^{C \times \mu}$ is the "error-location matrix", $r$ in $\mathbb{F}_{q}^{C \times k N}$ is the "message matrix", and $\hat{E}$ in $\mathbb{F}_{q}^{\delta \times k N}$ is the "known error value" (and its rank is denoted $\delta$ ).

2) Let $X=\left[\begin{array}{l}X_{1} \\ M_{2}^{f}\end{array}\right]$ and $e=r-X$ and $\tau=$ $\operatorname{rank}\left[\begin{array}{ll}\hat{L} & e \\ 0 & \hat{E}\end{array}\right]$. Then $2 \tau-\mu-\delta$ is no more than

\footnotetext{
${ }^{5}$ ) is from Prop. 7, 2) from Thm. 9, and 3) from Prop. 10 in [5].
} 
$d_{S}\left(\left[\begin{array}{ll}T_{R R E} & M_{R R E}\end{array}\right],\left[\begin{array}{ll}I_{C} & X\end{array}\right]\right)$, i.e., the row-space

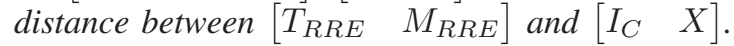

3) There exist $\tau$ column vectors $\mathbf{L}_{1}, \mathbf{L}_{2}, \ldots, \mathbf{L}_{\tau} \in \mathbb{F}_{q}^{C}$ and $\tau$ row vectors $\mathbf{E}_{1}, \mathbf{E}_{2}, \ldots, \mathbf{E}_{\tau} \in \mathbb{F}_{q}^{1 \times k N}$ such that $e=\sum_{i \in[1, \tau]} \mathbf{L}_{i} \mathbf{E}_{i}$. In particular, $\mathbf{L}_{1}, \mathbf{L}_{2}, \ldots, \mathbf{L}_{\mu}$ are the columns of $L$, and $\mathbf{E}_{\mu+1}, \mathbf{E}_{\mu+2}, \ldots, \mathbf{E}_{\mu+\delta}$ are the rows of $\hat{E}$.

Recall that the subscript $d$ stands for the last $N$ rows of any matrix/vector. Then we show the following for our scheme.

Lemma 3. 1) Matrix $e_{d}=r_{d}-M_{2}^{f}$ can be expressed as $e_{d}=\sum_{i \in 1,2, \ldots, \tau}\left(\mathbf{L}_{i}\right)_{d} \mathbf{E}_{i}$, where $\left(\mathbf{L}_{1}\right)_{d},\left(\mathbf{L}_{2}\right)_{d}, \ldots,\left(\mathbf{L}_{\mu}\right)_{d}$ are the columns of $\hat{L}_{d}$ and $\mathbf{E}_{\mu+1}, \mathbf{E}_{\mu+2}, \ldots, \mathbf{E}_{\mu+\delta}$ are the rows of $\hat{E}$.

2) With probability at least $1-|\mathcal{E}| / p, 2 \tau-\mu-\delta \leq 2 z$

Proof: 1) It is a direct corollary from Proposition 2.3.

2) Using Proposition 2.2 it suffices to prove with probability at least $1-|\mathcal{E}| / p, d_{S}\left(\left[\begin{array}{ll}T_{R R E} & M_{R R E}\end{array}\right],\left[\begin{array}{ll}I_{C} & X\end{array}\right]\right) \leq 2 z$.

As shown in the proof of Lemma 1, the columns of $E_{3}^{f}$ are in the column space of $E_{3}$ (and then of $E$ ) over $\mathbb{F}_{q}$. Thus $\left[\begin{array}{lll}E_{1} & E_{2} & E_{3}^{f}\end{array}\right]$ and therefore $\left[\begin{array}{lll}E_{1} G_{1} & E_{2} & E_{3}^{f}\end{array}\right]$ has rank at most equal to $z$ over $\mathbb{F}_{q}$. Using Proposition 1 and (5), $d_{S}\left(Y_{a},\left[\begin{array}{ccc}T_{1} G_{1} & T_{2} & A^{f}\end{array}\right]\right)$ is no more

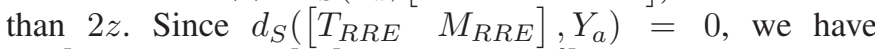
$d_{S}\left(\left[\begin{array}{cc}T_{R R E} & M_{R R E}\end{array}\right],\left[\begin{array}{lll}T_{1} G_{1} & T_{2} & A^{f}\end{array}\right]\right) \leq 2 z$.

Using Lemma 2 , matrix $D$ is invertible with probability at least $1-|\mathcal{E}| / p$, so $\left[\begin{array}{ll}I_{C} & X\end{array}\right]$ has zero rowspace distance from $\left[\begin{array}{ll}D & D X\end{array}\right]=\left[\begin{array}{lll}T_{1} G_{1} & T_{2} & A^{f}\end{array}\right]$. Thus $d_{S}\left(\left[\begin{array}{ll}T_{R R E} & M_{R R E}\end{array}\right],\left[\begin{array}{ll}I_{C} & X\end{array}\right]\right) \leq 2 z$.

In the end combining Lemma 3 and Theorem 1 the receiver $\mathcal{R}$ can take $\left(\hat{L}_{d}, \hat{E}, r\right)$ as the input for the Gabidulin decoding algorithm and decode $X_{2}$ correctly.

Stage 2: Decoding $X_{1}$ : From (4) the receiver gets $Y=$ $\left[\begin{array}{lll}T_{1}+E_{1} & T_{2}+E_{2} & A+E_{3}\end{array}\right]$, computes $\left(T_{2}+E_{2}\right) M_{2}$, and then subtracts matrix $\left[\begin{array}{lll}O & \left(T_{2}+E_{2}\right) & \left(T_{2}+E_{2}\right) M_{2}\end{array}\right]$ from $Y$. The resulting matrix has $N$ zero columns in the middle (column $n+1$ to column $n+N)$ ). Disregarding these we get:

$$
Y^{\prime}=\left[\begin{array}{ll}
T_{1} & T_{1} M_{1}
\end{array}\right]+\left[\begin{array}{ll}
E_{1} & E_{3}-E_{2} M_{2}
\end{array}\right] .
$$

The new error matrix $E^{\prime}=\left[\begin{array}{ll}E_{1} & E_{3}-E_{2} M_{2}\end{array}\right]$ has rank at most $z$ over $\mathbb{F}_{p}$ since the columns of $E^{\prime}$ are simply linear combinations of columns of $E$ whose rank is at most $z$. Therefore the problem degenerates into a single source problem and receiver $\mathcal{R}$ can decode $X_{1}$ with probability at least $1-|\mathcal{E}| / p$ by following the approach in [5].

Summarizing the above decoding scheme for $X_{1}$ and $X_{2}$, we have the main result of this work.

Theorem 3. A non-coherent receiver $\mathcal{R}$ can efficiently decode both $X_{1}$ and $X_{2}$ correctly with probability at least $1-2|\mathcal{E}| / p$.

\section{A. Complexity discussion}

For both coherent and non-coherent cases the computational complexity of Gabidulin encoding and decoding of two source messages is dominated by the decoding of $X_{2}$, which requires $\mathcal{O}(n N C \ell \log (n N))$ operations over $\mathbb{F}_{p}$ (see [5]).
To generalize our technique to more sources, consider a network with $s$ sources $\mathcal{S}_{1}, \mathcal{S}_{2}, \ldots, \mathcal{S}_{s}$. Let $R_{i}$ be the rate of $\mathcal{S}_{i}$ and $n_{i}=R_{i}+2 z$ for each $i \in[1, s]$. A straightforward generalization uses the multiple field-extension technique so that $\mathcal{S}_{i}$ uses the generator matrix over finite field of size $p^{n_{1} n_{2} \ldots n_{i}}$. In the end the packet length must be at least $n_{g}=n_{1} n_{2} \ldots n_{s}$, resulting in a decoding complexity $\mathcal{O}\left(C n_{g}^{2} \log \left(n_{g}\right)\right)$ increasing exponentially in the number of sources $s$. Thus the multiple field-extension technique works in polynomial time only for a fixed number of sources.

\section{ACKNOWLEDGMENTS}

The work of Hongyi Yao was supported by National Natural Science Foundation of China Grant 60553001, the National Basic Research Program of China Grant 2007CB807900 and 2007CB807901. The work of Theodoros K. Dikaliotis and Tracey Ho was supported by NSF grant CNS-0905615. The work of Sidharth Jaggi was supported by RGC GRF grants 412608, 411008, and 411209, a grant from the University Grants Committee of the Hong Kong Special Administrative Region, China (Project No. AoE/E-02/08), CUHK MoEMicrosoft Key Laboratory of Humancentric Computing and Interface Technologies, and a gift grant from Cisco.

\section{REFERENCES}

[1] R. Ahlswede, N. Cai, S.-Y. Li, and R. Yeung, "Network information flow," IEEE Transactions on Information Theory, vol. 46, no. 4, pp. 1204-1216, July 2000.

[2] M. Siavoshani, C. Fragouli, and S. Diggavi, "Code construction for multiple sources network coding," in Proc. of the MobiHoc, 2009.

[3] N. Cai and R. W. Yeung, "Network coding and error correction," in Proc. of 2002 IEEE Information Theory Workshop (ITW), 2002.

[4] S. Jaggi, M. Langberg, S. Katti, T. Ho, D. Katabi, and M. Médard, "Resilient network coding in the presence of byzantine adversaries," in Proc. IEEE INFOCOM 2007, 2007.

[5] D. Silva, F. Kschischang, and R. Koetter, "A rank-metric approach to error control in random network coding," IEEE Transactions on Information Theory, vol. 54, no. 9, pp. 3951-3967, Sept. 2008.

[6] D. Silva and F. R. Kschischang, "On metrics for error correction in network coding," in Proc. of 46th Annual Allerton Conf. on Commun., Control, and Computing, 2008.

[7] T. Ho, M. Médard, R. Koetter, D. R. Karger, M. Effros, J. Shi, and B. Leong, "A random linear network coding approach to multicast," IEEE Transactions on Information Theory, vol. 52, no. 10, pp. 44134430, 2006.

[8] M. Siavoshani, C. Fragouli, and S. Diggavi, "Noncoherent multisource network coding," in Proc. of the IEEE ISIT, 2008.

[9] M. Siavoshani, S. Mohajer, C. Fragouli, and S. Diggavi, "On the capacity of non-coherent network coding," in Proc. of the IEEE ISIT, 2009.

[10] S. Vyetrenko, T. Ho, M. Effros, J. Kliewer, and E. Erez, "Rate regions for coherent and noncoherent multisource network error correction," in Proc. of ISIT 2009, 2009.

[11] T. Ho, M. Médard, J. Shi, M. Effros, and D. R. Karger, "On randomized network coding," in Proc. of Allerton 2003, 2003.

[12] M. Artin, Algebra. New Jersey: Prentice Hall, 1991.

[13] R. Kötter and F. R. Kschischang, "Coding for errors and erasures in random network coding," IEEE Transactions on Information Theory, vol. 54, no. 8, pp. 3579-3591, Aug. 2008.

[14] E. M. Gabidulin, "Theory of codes with maximum rank distance," Probl. Peredachi Inf., vol. 21, no. 1, pp. 3-16, 1985.

[15] S. Jaggi, P. Sanders, P. A. Chou, M. Effros, S. Egner, K. Jain, and L. Tolhuizen, "Polynomial time algorithms for multicast network code construction," IEEE Transactions on Information Theory, vol. 51, no. 6, pp. 1973-1982, 2003.

[16] Z. Richard, Effective Polynomial Computation. Springer, 1993. 